\title{
金属催化非晶碳转化制备石墨烯方法的研究进展
}

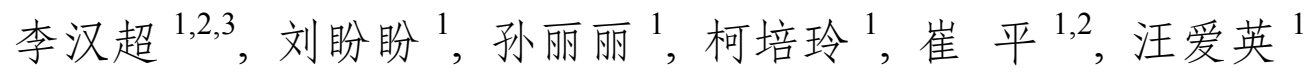

(1. 中国科学院宁波材料技术与工程研究所, 中国科学院海洋新材料与应用技术重点实验室, 浙江省海洋材料与 防护技术重点实验室, 宁波 $315201 ; 2$. 上海科技大学 物质科学与技术学院, 上海 201210; 3. 中国科学院大学, 北京 100049)

摘 要: 石墨烯自 2004 年被首次发现以来，以其独特、优异的结构和特性引起了广泛关注。目前，石墨烯的制备已 取得了众多进展，但在大尺寸、高质量、宏量石墨烯可控制备上仍存在挑战，对制备技术仍需要进行更广泛地探索。 非晶碳与石墨烯互为碳的同素异形体，也可作为制备石墨烯的前驱体，近些年利用非晶碳制备石墨烯的新颖方法 引起了研究学者的兴趣。本文系统论述了利用非晶碳作为固体碳源，通过金属催化制备大尺寸高质量石墨烯的技术 优势，并着重从金属种类、退火温度、碳源及金属含量比例等方面对石墨烯生成质量的影响进行了阐述。最后，总 结了该方法生长石墨烯的机理, 并对未来的发展方向进行了展望。

关 键 词: 非晶碳; 石墨烯; 固体碳源; 金属催化

中图分类号: TQ127 文献标识码: A

\section{Recent Development of the Transformation from Amorphous Carbon to Graphene Method via Metal Catalyst}

\author{
LI Han-Chao ${ }^{1,2,3}$, LIU Pan-Pan ${ }^{1}$, SUN Li-Li ${ }^{1}$, KE Pei-Ling ${ }^{1}$, CUI Ping ${ }^{1,2}$, WANG Ai-Ying ${ }^{1}$
}

(1. Key Laboratory of Marine Materials and Related Technologies, Zhejiang Key Laboratory of Marine Materials and Protective Technologies, Ningbo Institute of Materials Technology and Engineering, Chinese Academy of Sciences, Ningbo 315201, China; 2. School of Physical Science and Technology, ShanghaiTech University, Shanghai 201210, China; 3. University of Chinese Academy of Sciences, Beijing 100049, China)

\begin{abstract}
Graphene has drawn wide attention owing to its excellent properties since the discovery in 2004. There are many methods to produce graphene so far but many challenges still exist in producing scalable and high quality graphene. Thus, preparation of graphene should be further explored. Amorphous carbon and graphene are two allotropes of carbon. As one kind of solid carbon source, amorphous carbon could also be used to produce graphene. The preparation of graphene from amorphous carbon via metal catalyst has aroused much interest. Here, the advantages of using amorphous carbon as solid carbon source, to get high quality and large size graphene via metal catalyst in recent years are summarized. Several influence factors, such as metal sort, annealing temperature, the ratio of amorphous carbon to metal, have been discussed elaborately. Meanwhile, transformation mechanism from amorphous carbon to graphene has been reviewed and summarized simultaneously. Finally, the prospect of this novel fabrication method for graphene was put forward.
\end{abstract}

Key words: amorphous carbon; graphene; solid carbon source; metal catalyst

收稿日期：2017-11-09; 收到修改稿日期：2017-12-25

基金项目：国家自然科学基金(51371187); 浙江省重点研发计划(2017C01001); 浙江省公益项目(2016C31121) National Natural Science Foundation of China (51371187); Zhejiang Key Research and Development Program (2017C01001); Public Projects of Zhejiang Province (2016C31121)

作者简介：李汉超(1993-), 男，博士研究生. E-mail: lihanchao@nimte.ac.cn

通讯作者: 汪爱英, 研究员. E-mail: aywang@nimte.ac.cn 
碳元素(C)在自然界中广泛存在, 而且有多种 同素异形体，具备诸多优异性质，在国民经济、军事 和人类生活中发挥着重要作用。科学家们已相继发 现并合成了多种新型碳材料, 例如, 1955 年高温高 压法制备了金刚石 ${ }^{[1]}, 1971$ 年离子束沉积制备了非

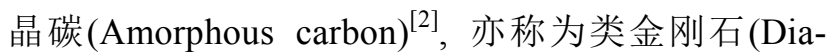
mond like carbon); 1985 年激光气化蒸发石墨制备了 富勒烯 ${ }^{[3]}, 1991$ 年采用化学气相沉积 (Chemical Vapor Deposition, CVD)合成了碳纳米管 ${ }^{[4]}, 2004$ 年利 用机械剥离石墨发现的石墨烯 ${ }^{[5]}$, 以及 2010 年我国 科学家利用六炔基苯在铜片的催化作用下发生偶联 反应，通过化学方法合成的石墨炔薄膜 ${ }^{[6]}$ 。

其中, 石墨烯是一种完全由 $\mathrm{sp}^{2}$ 杂化 $\mathrm{C}$ 原子形 成的六边形蜂窝状二维晶体, 仅有一个原子层厚度, 这种独特的二维结构使得石墨烯具有许多三维材料 无法媲美的优异性能, 如优异的力学性能 ${ }^{[7]}$ : 杨氏 模量高达 $1 \mathrm{TPa}$, 断裂强度 $130 \mathrm{GPa}$; 光学性能 ${ }^{[8]}$, 单 层石墨烯的透光性高达 $97.7 \%$; 高热导率 ${ }^{[9]}$ ，单层石 墨烯导热率高达 $5000 \mathrm{~W} / \mathrm{mK}$, 是 $\mathrm{Cu}$ 的十几倍; 优异的 电学性能 ${ }^{[10]}$ : 载流子迁移率可达到 $200000 \mathrm{~cm}^{2} /(\mathrm{V} \cdot \mathrm{s})$ 。 这些特性使得石墨烯在生物医学 ${ }^{[11]}$ 、超级电容器 ${ }^{[12] 、}$ 太阳能电池 ${ }^{[13]}$ 和高频场效应晶体管 ${ }^{[14]}$ 等领域具有 广阔的应用前景。

目前，已经发展了很多石墨烯的制备方法，包

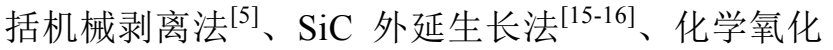
还原法 ${ }^{[17-18]}$ 、化学气相沉积法 $(C V D)^{[19-22]}$ 等。从合 成石墨烯质量、工艺可控性和成本等角度来看, 这 些方法都存在各自的局限性。

2010 年, Sun 等 ${ }^{[23]}$ 利用高分子聚合物(PMMA) 作为固体碳源, 成功制备了高质量的石墨烯。相比 于使用 $\mathrm{CH}_{4} 、 \mathrm{C}_{2} \mathrm{H}_{2}$ 等气体碳源, 利用固体碳源 ${ }^{[24-25]}$ 可在更低温度下制备大面积、高质量且层数可控的 石墨烯, 因此越来越受到研究人员的重视。非晶碳 作为一种固体碳源, 引起了广泛关注。非晶碳 (Amorphous carbon)是一种由 $\mathrm{sp}^{2}$ 杂化 $\mathrm{C}$ 原子和 $\mathrm{sp}^{3}$ 杂化 $\mathrm{C}$ 原子组成的亚稳态材料 ${ }^{[26]}$, 具有优异的减摩 抗磨性能、低的介电常数、宽带隙以及良好的光学 透过性, 被广泛应用于航空航天、机械、电子等领 域 ${ }^{[27-29]}$ 。自上世纪 70 年代成功运用离子束沉积技术 获得非晶碳以来 ${ }^{[2]}$, 非晶碳的制备方法越来越成熟, 更趋简易, 可由各种物理气相沉积(Physical Vapor Deposition, PVD)或 CVD 方法在低温下大面积制 备。此外, 非晶碳与石墨烯同属碳的同素异形体, 作 为一种含有石墨相 $\mathrm{sp}^{2}$ 和金刚石相 $\mathrm{sp}^{3}$ 的新型碳材料, 一直是碳材料领域的研究热点。利用非晶碳热退火 为直接在绝缘基底上制备石墨烯提供了可能, 具备
巨大的应用价值。

本文详细介绍了近年来以非晶碳为固体碳源制 备石墨烯取得的研究进展，重点阐述了制备石墨烯 的影响因素以及相关生长机理, 并对非晶碳转变为 石墨烯的研究方向进行了展望。

\section{1 催化方法}

非晶碳转化为石墨烯均需通过加热方式获得, 一般采用激光辐照加热和常规退火热处理两种方法。

Koshida 等 $^{[30]}$ 利用激光辐照法在绝缘基底上直 接合成了多层石墨烯，他们利用激光加热金属 $\mathrm{Ni}$, 使得非晶碳扩散进入 $\mathrm{Ni}$ 中, 在 $\mathrm{Ni}$ 金属熟化收缩作 用下，碳原子最终以石墨烯的形式重新析出，如图 1 所示。他们还发现, 在实验中引入氢气, 可以提高石 墨烯的生成质量, 拉曼结果显示, 石墨烯的 $\mathrm{D}$ 和 $\mathrm{G}$ 峰明显分化, 且 2D 峰明显增强, 如图 2 所示, 但其 未对石墨烯转化作用机理进行阐述。

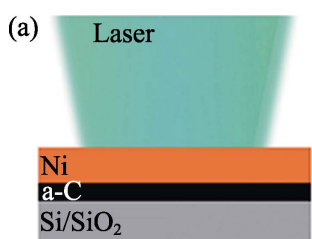

(c)

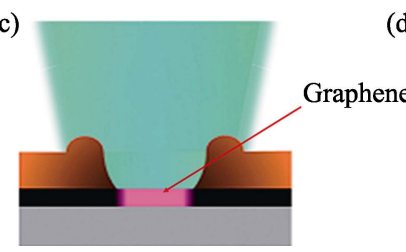

(b)

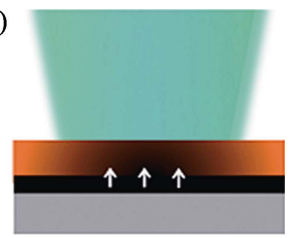

(d)

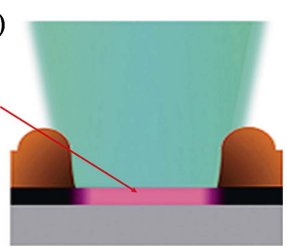

图 1 激光照射下石墨烯形成原理图 ${ }^{[30]}$

Fig. 1 Schematic of graphene synthesis mechanism ${ }^{[30]}$ (a) Local annealing of the Ni layer by laser irradiation; (b) Dissolution of a-C in the Ni layer; (c) Aggregation and retraction of the Ni layer; (d) Direct graphene synthesis on a $\mathrm{SiO}_{2}$ surface

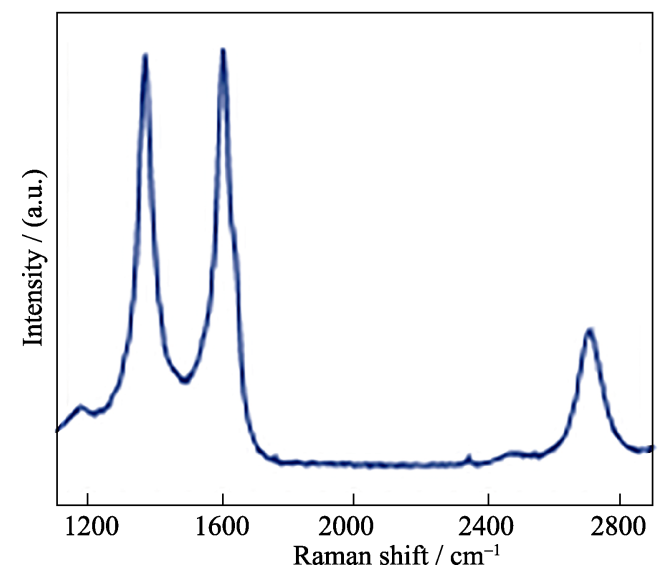

图 2 基底 $200^{\circ} \mathrm{C}$ 、氢气气氛下激光辐照样品的拉曼光谱图 ${ }^{[30]}$ Fig. 2 Raman spectrum for a sample irradiated with a laser in $\mathrm{H}_{2}$ atmosphere at a substrate temperature of $200^{\circ} \mathrm{C}^{[30]}$ 
利用激光辐照加热使非晶碳转化合成石墨烯的 研究目前较少, 大多加热方式还是常规退火热处理。

退火热处理(Thermal Annealing- TA) 是实现 非晶碳转化制备石墨烯的一种主要方法。该方法是 在基底上沉积金属层和非晶碳层后, 再进行热退火, 使非晶碳转变生成石墨烯。根据金属层和非晶碳层 的沉积次序, 可分为非晶碳沉积在催化金属下方和 上方两种三明治方式(图 3 和图 4), 这两种方式经热 处理后均能够获得石墨烯。如 Zheng 等 ${ }^{[31]}$ 将金属 $\mathrm{Ni}$ 层沉积在非晶碳上, 经过热退火, 可在 $\mathrm{Ni}$ 表面获 得石墨烯, 其拉曼数据如图 5 所示。而 Seo 等 ${ }^{[32}$ 将 非晶碳膜沉积在金属 $\mathrm{Ni}$ 层上, 经过热退火在 $\mathrm{Ni}$ 膜 表面亦得到石墨烯。

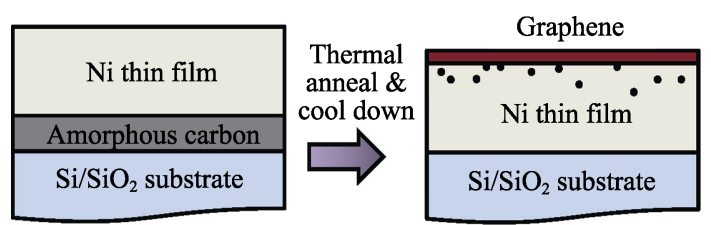

图 3 热退火方法金属催化非晶碳转化石墨烯示意图 ${ }^{[31]}$

Fig. 3 Process schematic for the metal-catalyzed crystallization of a-C to graphene by thermal annealing ${ }^{[31]}$

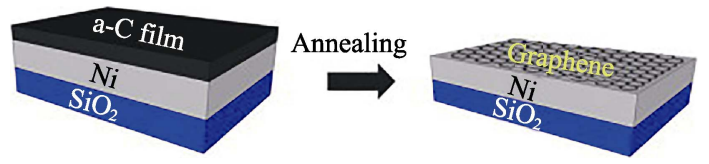

图 4 石墨烯生长方法示意图 ${ }^{[32]}$

Fig. 4 Schematic illumination of the graphene growth meth$\mathrm{od}^{[32]}$

由于研究人员各自的实验条件不同, 对于上述 两种结构制备得到的石墨烯质量各有差异, 如表 1 汇总所示。

研究人员制备前驱体非晶碳的方法多样，包括
电子束蒸镀(Electron Beam Evaporation, EBE)、过滤 真空电弧沉积(Filtered Vacuum Arc Deposition, FVAD)、脉冲等离子体电弧沉积(Pulse Arc Plasma Deposition, PAPD)、激光烧蚀(Laser Ablation, LA), 聚焦离子束化学气相沉积(Focused-Ion-Beam Chemical
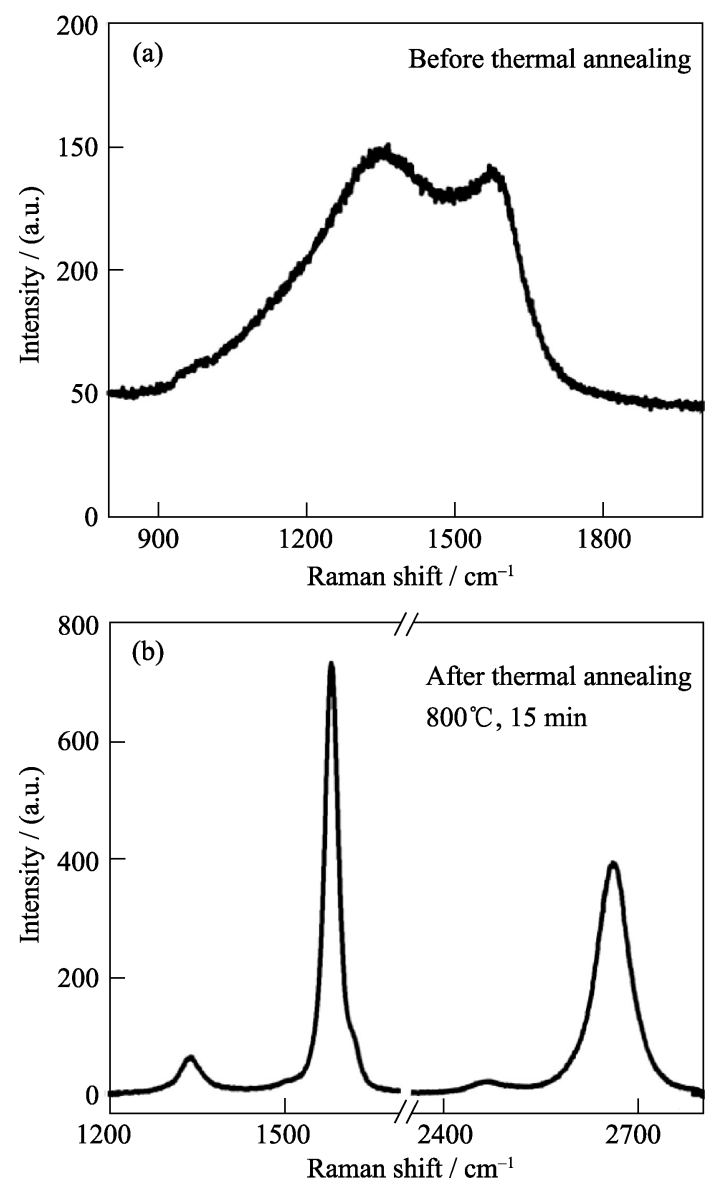

图 5 (a) a-C(40 nm)和(b)Ni 催化结晶后得到的石墨烯( $\mathrm{Ni}$ 厚 度 300 nm) 的 Raman 光谱图, 激光发射波长 $632.8 \mathrm{~nm}^{[31]}$

Fig. 5 Raman spectra of (a) a-C (40 nm) and (b) the resulting graphene layer after Ni-catalyzed crystallization (Ni thickness $\sim 300 \mathrm{~nm}$ ) with excitation laser wavelength $632.8 \mathrm{~nm}^{[31]}$

表 1 退火热处理非晶碳转化石墨烯的实验参数及结果

Table 1 Parameters and results of thermal annealing for the experiment of transformation of a-C to graphene

\begin{tabular}{cccccccccccccc}
\hline a-C method & $T /{ }^{\circ} \mathrm{C}$ & $\begin{array}{c}\text { Annealing } \\
\text { time/min }\end{array}$ & Gas & Catalyst & Pressure/Pa & $\begin{array}{c}\text { Graphene } \\
\text { morphology }\end{array}$ & \multicolumn{2}{c}{ Raman } & $I_{\mathrm{D}} / I_{\mathrm{G}}$ & $I_{2 \mathrm{D}} / I_{\mathrm{G}}$ & Ref. \\
\hline EBE & $650-950$ & 15 & $\mathrm{Ar}$ & $\mathrm{Ni}, \mathrm{Co}$ & 226 & Single, double layer & 0.09 & - & {$[31]$} \\
FVAD & $600-1000$ & 5 & - & $\mathrm{Ni}$ & - & Few layer & - & - & {$[32]$} \\
PAPD & $700-1000$ & 5 & $\mathrm{~N}_{2}$ & $\mathrm{Co}, \mathrm{Ni}$ & - & Multilayer & 0.59 & 1 & {$[35]$} \\
LA & 1000 & 30 & - & $\mathrm{Ga}$ & $1.33 \times 10^{-2}$ & Few layer & - & - & {$[36]$} \\
FIB-CVD & $900-1100$ & 30,60 & - & $\mathrm{Ga}$ & - & Three, four layer & - & 0.84 & {$[37]$} \\
PLD & 800 & 5 & $\mathrm{Ar}$ & $\mathrm{Co}$ & 0.1 & Single, double layer & 2.5 & $0.67-1.43$ & {$[39]$} \\
MS & $750-800$ & $5-10$ & - & $\mathrm{Co}, \mathrm{Ni}$ & $3.0 \times 10^{-4}$ & Single, double layer & - & 1.43 & {$[40]$} \\
DCMS & 1100 & 2 & $\mathrm{Ar}$ & $\mathrm{Ni}$ & 0.266 & Single, double layer & - & 2.5 & {$[46]$} \\
PAPD & 900 & 5 & $\mathrm{~N}_{2}$ & $\mathrm{Ni}$ & - & Multilayer & - & - \\
DCMS & 900 & 30 & - & $\mathrm{Ni}$ & $2.66 \times 10^{-4}$ & Single, double layer & 0.03 & 4.8 & {$[42]$} \\
\hline
\end{tabular}


Vapor Deposition, FIB-CVD)、脉冲激光沉积(Pulsed Lased Deposition, PLD)、磁控溅射(Magnetron Sputtering, MS)和直流磁控溅射 (Direct Current Magnetron Sputtering, DCMS)等等, 但经过热处理得到 的石墨烯差异并不大, 均可制备多层、少层甚至单 层的石墨烯，表明后续试验中，热处理过程、金属的 种类、金属/碳膜比例、退火温度、时间、升/降温 速率和气氛等实验参数对生成石墨烯的质量具有重 要影响。

\section{1 金属种类}

不同金属催化非晶碳转化石墨烯的能力不同, 导致生成石墨烯质量有所区别。Rodríguez 等 ${ }^{[25]}$ 在 $600^{\circ} \mathrm{C}$ 以上退火时, 采用 $\mathrm{Cu} 、 \mathrm{Fe} 、 \mathrm{Ni} 、 \mathrm{Co}$ 四种典型 金属作为催化剂, 发现 $\mathrm{Fe} 、 \mathrm{Ni} 、 \mathrm{Co}$ 三者作为催化剂 时均生成了石墨烯, 而 $\mathrm{Cu}$ 作为催化剂则无法得到 石墨烯。这是由于 $\mathrm{C}$ 原子在 $\mathrm{Cu}$ 中的溶解度低, 石 墨烯无法进行形核生长。Peng 等 ${ }^{[33]}$ 也发现, 当 $\mathrm{Ni}$ 作为催化剂, 利用 PMMA 经过退火后形成了石墨烯, 但采用 $\mathrm{Cu}$ 作为催化剂时, 检测不到石墨烯的拉曼 信号峰(图 6)。 $\mathrm{Li}$ 等 ${ }^{[34]}$ 进一步研究表明这主要是由 于 $\mathrm{C}$ 原子在 $\mathrm{Cu}$ 和 $\mathrm{Ni}$ 中的溶解度不同, 导致生成石 墨烯的机理不同。对于 $\mathrm{Cu}$ 等溶碳量较低的金属而 言, 其生长机理为表面催化生长。高温下, 碳源在 $\mathrm{Cu}$ 的表面裂解生成 $\mathrm{C}$ 原子吸附在金属表面, 之后形 核生长成石墨烯片, 再长大合并得到连续的大面积 石墨烯薄膜。而对于 $\mathrm{Ni}$ 等高溶碳量的金属而言, 其 生长机理为溶解析出。高温下, 碳源裂解生成 $\mathrm{C}$ 原 子溶解渗入金属基体内, 降温时 $\mathrm{C}$ 原子再从金属内 部重新析出, 形核长大形成石墨烯。

正是由于 $\mathrm{C}$ 在不同金属中的溶解度不同, 相同 条件下生成的石墨烯层数也有差异 ${ }^{[35]}$ 。如 Rodríguez 等 ${ }^{[25]}$ 研究发现, 由于 $\mathrm{C}$ 在 $\mathrm{Ni} 、 \mathrm{Co}$ 中的溶解度低于 在 $\mathrm{Fe}$ 中溶解度, 利用 $\mathrm{Ni} 、 \mathrm{Co}$ 作为催化剂得到了单 层和少层石墨烯, 而利用 $\mathrm{Fe}$ 作为催化剂则得到多层 石墨烯。

还有研究者将 $\mathrm{Ga}$ 作为催化剂研究其催化性能。 Fujita 等 ${ }^{[36]}$ 利用液体 $\mathrm{Ga}$ 在 $700^{\circ} \mathrm{C} 、 1.33 \times 10^{-2} \mathrm{~Pa}$ 条 件下退火 $30 \mathrm{~min}$, 使非晶碳转变成石墨烯。TEM 证 实石墨烯为多层石墨烯, 且存在非晶碳夹层; 拉曼 结果显示出很强的 D 峰, 表明存在大量缺陷。另外, 他们发现 $\mathrm{Ga}$ 的催化作用仅限于非晶碳与 $\mathrm{Ga}$ 的界面 处。Hatakeyama 等 ${ }^{[37]}$ 同样利用 $\mathrm{Ga}$ 作为催化剂, 分 别在 900、1000、 $1100^{\circ} \mathrm{C}$ 真空环境中进行实验，发现 $\mathrm{Ga}$ 倾向于形成液滴状, 因此 $\mathrm{Ga}$ 的催化性能局限于 其与非晶碳所接触的球面。为了增大金属液滴与非 晶碳的接触面积, 有研究者 ${ }^{[38]}$ 通过掺杂 $\mathrm{Cu}$ 使其与
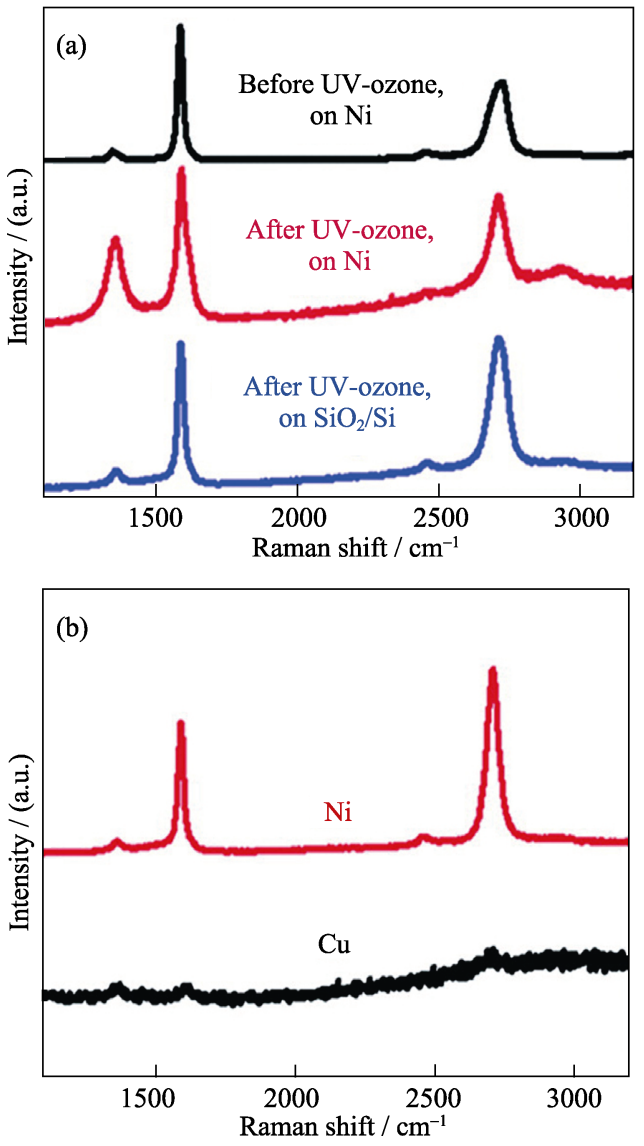

图 6 不同生长条件下拉曼分析结果 ${ }^{[33]}$

Fig. 6 Raman spectroscopic analysis of graphene from different growth conditions ${ }^{[33]}$

(a) Raman spectra of graphene on the top of the nickel layer before and after UV-ozone exposure, and graphene on the substrate after UV-ozone exposure and nickel removal; (b) Raman spectra of PMMA-derived graphene by different metal catalysts

$\mathrm{Ga}$ 形成合金，降低其表面张力，使得合金倾向于形 成平面液面，最终得到大面积高质量的石墨烯。

由此可见, 正是由于 $\mathrm{C}$ 在不同金属中的溶解度 不同, 造成生成的石墨烯的层数和质量不同。C 在 $\mathrm{Cu}$ 中的溶解度非常低, 无法通过热退火得到石墨烯; $\mathrm{C}$ 在 Co、Ni 中的溶解度较好, 可以得到石墨烯, 通 过合理调控其它条件，还可以制备少层甚至单层石 墨烯; C 在 $\mathrm{Fe}$ 中的溶解度非常高, 虽然可以制备出 石墨烯, 但是层数很难控制, 往往是多层石墨烯。

\section{2 退火温度}

退火温度对石墨烯的转变影响显著。Zheng 等 ${ }^{[31]}$ 研究表明, 在非晶碳厚度为 $40 \mathrm{~nm}$ 时, 选用 Co 和 $\mathrm{Ni}$ 金属层均可在 $650 \sim 850^{\circ} \mathrm{C}$ 退火处理后得到石墨烯, 且在 $750^{\circ} \mathrm{C}$ 退火得到的石墨烯结果最佳, 得到近单 层的石墨烯。Seo 等 ${ }^{[32]}$ 选用 $\mathrm{Ni}$ 作为催化剂, $15 \mathrm{~nm}$ 厚非晶碳为原料时, 经过 $600^{\circ} \mathrm{C}$ 退火并没有检测到 石墨烯的 $2 \mathrm{D}$ 特征峰; 当退火温度为 $800^{\circ} \mathrm{C}$ 时, 可制 备出多层石墨烯; 而退火温度升高至 $1000^{\circ} \mathrm{C}$ 时, $2 \mathrm{D}$ 
峰宽化，石墨烯的质量下降。Hirano 等 ${ }^{[39]}$ 则利用 Co 作为催化剂, 在 $\mathrm{Ar}$ 条件下, 对比研究了 $600^{\circ} \mathrm{C}$ 和 $800^{\circ} \mathrm{C}$ 退火的实验结果, 发现 $800^{\circ} \mathrm{C}$ 退火时, $\mathrm{Co}$ 的催 化性能更优异, 获得石墨烯质量好。同样, Orofeo 等 ${ }^{[40]}$ 也以 $\mathrm{Co}$ 和 $\mathrm{Ni}$ 作为催化剂, 厚度为 $1 \mathrm{~nm}$ 的非 晶碳在 $3.0 \times 10^{-4} \mathrm{~Pa}$ 气压下, $750 \sim 800^{\circ} \mathrm{C}$ 退火温度范围 内得到的石墨烯质量最佳, 而 $500^{\circ} \mathrm{C}$ 退火得到的石 墨烯均一性较差。上述结果表明, 以金属 $\mathrm{Co}$ 和 $\mathrm{Ni}$ 为催化剂时, 均存在优化的退火温度区间(600 $800^{\circ} \mathrm{C}$ ), 并且最佳退火温度在 $750^{\circ} \mathrm{C}$ 左右。为了进一 步研究退火温度对非晶碳转化生成石墨烯过程的影 响, Saenger 等 ${ }^{[41]}$ 利用 XRD 进行分析, 结果显示以 金属 $\mathrm{Ni}$ 作为催化剂时, 非晶碳转化石墨烯的温度在 $640 \sim 730^{\circ} \mathrm{C}$; 在 $600^{\circ} \mathrm{C}$ 以上, 非晶碳会逐渐开始石墨 化; 而当温度高于 $950^{\circ} \mathrm{C}$ 时, 碳原子会再次溶进金 属 $\mathrm{Ni}$ 中, 进而使得到的石墨烯结构消失, 如图 7 所 示。与上述研究类似, 对于 $\mathrm{Ni}$ 体系来说, 的确存在 使非晶碳转化石墨烯质量最佳的温度区间。

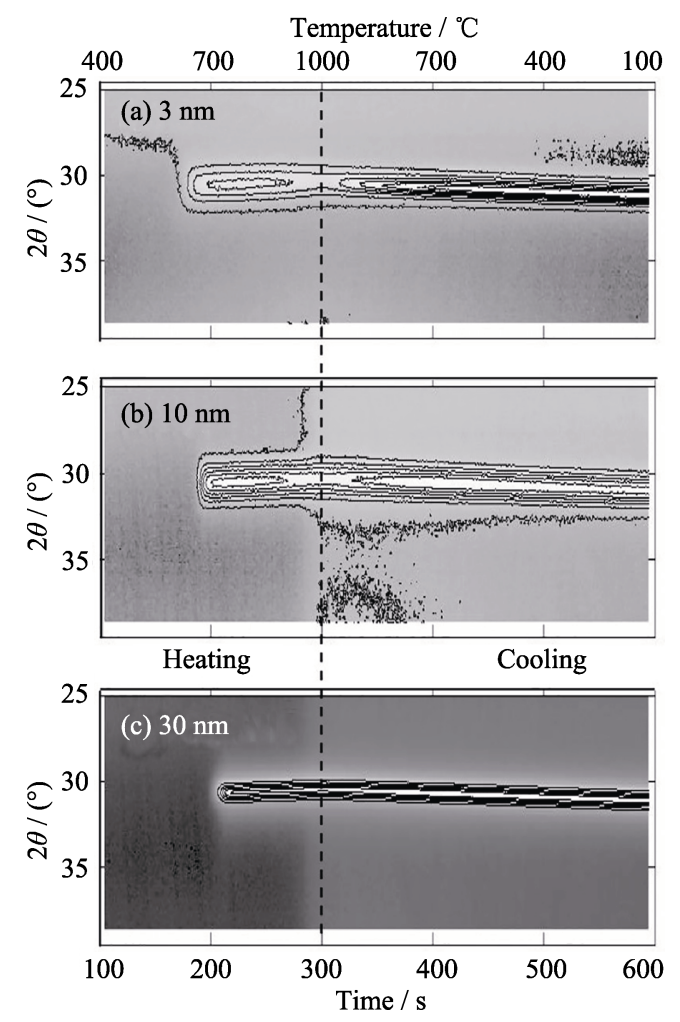

图 7 不同厚度 $\mathrm{Si} / \mathrm{SiO}_{2} / \mathrm{a}-\mathrm{C} / \mathrm{Ni}(100 \mathrm{~nm})$ 样品在 $\mathrm{He}$ 气氛下加 热至 $1000^{\circ} \mathrm{C}$ 及冷却过程中原位 XRD 的(002)石墨峰强等高线 图, 变温速率为 $3^{\circ} \mathrm{C} / \mathrm{s}$, 厚度(a) $3 \mathrm{~nm} 、$ (b) $10 \mathrm{~nm}$ 和(c) $30 \mathrm{~nm}$ 。 不同厚度 $\mathrm{a}-\mathrm{C}$ 样品的等高线间隔不同 ${ }^{[41]}$

Fig. 7 Contour maps of in situ XRD results showing the 002 graphite peak in $\mathrm{Si} / \mathrm{SiO}_{2} / \mathrm{a}-\mathrm{C} / \mathrm{Ni}(100 \mathrm{~nm})$ samples heated to and cooled from $1000^{\circ} \mathrm{C}$ in $\mathrm{He}$ at a ramp rate of $3{ }^{\circ} \mathrm{C} / \mathrm{s}$ for a-C thicknesses of (a) $3 \mathrm{~nm}$, (b) $10 \mathrm{~nm}$, and (c) $30 \mathrm{~nm}$. The contour lines have a linear intensity spacing that is different for each a-C thickness ${ }^{[41]}$

\section{3 碳源与金属的含量比例}

在金属催化非晶碳转变的过程中, 非晶碳的含 量一直是研究者密切关注的问题。这主要是因为非 晶碳过多, 不易被完全催化; 而非晶碳太少, 生成 的石墨烯质量不可控。Seo 等 ${ }^{[32]}$ 分别采用 $1 、 5 、 15$ 、 $50 \mathrm{~nm}$ 的 $\mathrm{a}-\mathrm{C}$, 在 $\mathrm{Ni}$ 厚度为 $300 \mathrm{~nm}, 800^{\circ} \mathrm{C}$ 下退火 $5 \mathrm{~min}$, 在 $15 \mathrm{~nm}$ 厚的非晶碳体系中得到多层石墨烯, 但在其它碳膜厚度下，对应的拉曼谱图没有出现明 显的石墨烯 $2 \mathrm{D}$ 特征峰。Banno 等 ${ }^{[42]}$ 选用不同厚度 的 $\mathrm{Ni}$, 对 $5 \mathrm{~nm}$ 非晶碳在 $900^{\circ} \mathrm{C} 、 \mathrm{~N}_{2}$ 条件下退火 $5 \mathrm{~min}$ 发现, 当 $\mathrm{Ni}$ 厚度为 $35 \mathrm{~nm}$ 时, 可以得到少层石墨烯, 质量最好; 当 $\mathrm{Ni}$ 厚度为 10 和 $20 \mathrm{~nm}$ 时, 得到的石 墨烯均超过 10 层。 $\mathrm{Chu}$ 等 ${ }^{[43]}$ 在 $\mathrm{SiO}_{2}$ 上沉积一层 $\mathrm{Ni}$ 后放置在空气中，使得 $\mathrm{Ni}$ 表面自发形成一层很薄的 几个纳米厚度的镍碳化物以及镍碳氧化物层, 最后 在 $\mathrm{Ar} / \mathrm{N}_{2} 、 800 \sim 1000^{\circ} \mathrm{C}$ 退火 $0.5 \mathrm{~min}$ 得到接近单层的 石墨烯。另外, Orofeo 等 ${ }^{[40]}$ 通过引入超薄非晶碳 $(1 \mathrm{~nm})$, 分别利用 $\mathrm{Co}$ 和 $\mathrm{Ni}$ 为催化金属层, 在 750 $800^{\circ} \mathrm{C} 、 3.0 \times 10^{-4} \mathrm{~Pa}$ 压力下退火 5 10 min, 均得到 接近单层的石墨烯。这可能是由于碳源与金属含量 的比例适当, 所以非晶碳一旦转化成石墨烯, 质量 会较高。根据 Wenisch 等 ${ }^{[44]}$ 研究, $\mathrm{Ni}$ 在催化非晶碳 转化成石墨烯时, 开始阶段非晶碳会倾向于在与 $\mathrm{Ni}$ 平行的表面生成石墨烯，而当碳源过多时，即石墨 烯超过 1 2 $\mathrm{nm}$ 之后, $\mathrm{C}$ 原子就会垂直生长, 并且无 序排列。Anton 等 ${ }^{[45]}$ 实验结果也表明, 越接近 $\mathrm{Ni}$ 表 面的 C 原子越倾向于以石墨烯的形式平行排列。但 碳源的量并非越少越好, 因为 $\mathrm{C}$ 在 $\mathrm{Ni}$ 中有一定溶解 度, 并且 $\mathrm{C}$ 还有可能挥发 ${ }^{[42]}$ 。因此, 对于非晶碳和催 化金属组合体系，需要综合考虑上述因素才能得到 高质量的石墨烯。例如, Zheng 等 ${ }^{[31]}$ 利用不同厚度的 非晶碳, 当 $\mathrm{Ni}$ 为 $300 \mathrm{~nm}$, 在 $800^{\circ} \mathrm{C}$ 下进行退火 $15 \mathrm{~min}$, 得到非晶碳厚度与生成石墨烯厚度呈线性关系(图 8)。

以上说明生成的石墨烯层数受非晶碳和 $\mathrm{Ni}$ 厚 度组合配比的综合作用。Xiong 等 ${ }^{[46]}$ 利用这一点, $1100^{\circ} \mathrm{C}$ 直接在 $\mathrm{SiO}_{2}$ 基底得到了单层石墨烯。他们认 为该过程的主要影响因素是 $\mathrm{C}$ 与 $\mathrm{Ni}$ 形成了 $\mathrm{Ni}_{3} \mathrm{C}^{[47-48]} 。 \mathrm{Ni}_{3} \mathrm{C}$ 在高于 $400^{\circ} \mathrm{C}$ 时分解, 会造成 $\mathrm{Ni}$ 金 属在远低于其熔点时即可挥发, 使得在 $\mathrm{SiO}_{2}$ 基底上 直接得到单层石墨烯。此外, 作者还详细研究了非 晶碳厚度以及 $\mathrm{Ni}$ 金属层厚度与制备的石墨烯质量 的相关性, 如图 9, 证实只有在合适的 $\mathrm{Ni} 、 \mathrm{C}$ 厚度范 围才能得到单层高质量的石墨烯。

\section{4 其它因素}

此外, 还有一些因素也会对非晶碳转变生成的 石墨烯过程造成影响，例如金属催化剂表面的平整 

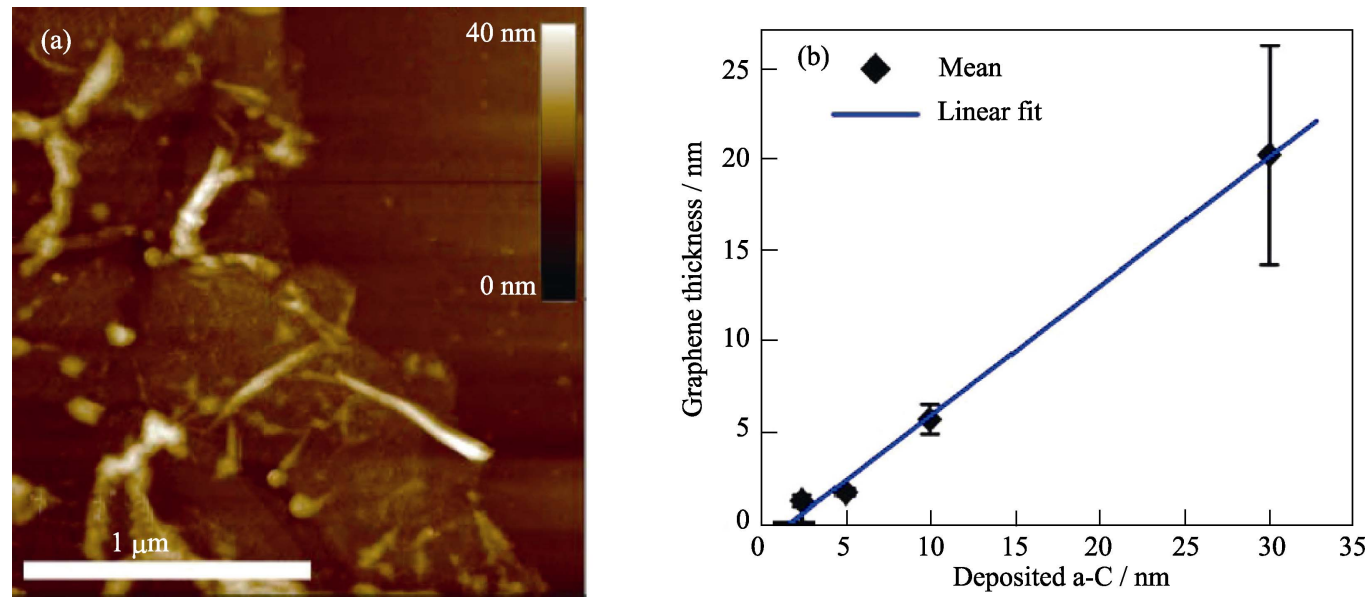

图 8 (a)转移到 $\mathrm{Si} / \mathrm{SiO}_{2}$ 后的石墨烯 AFM 照片和(b)石墨烯厚度与原始 a-C 厚度的关系 ${ }^{[31]}$

Fig. 8 (a) AFM image of a transferred graphene sheet on $\mathrm{Si} / \mathrm{SiO}_{2}$ substrate; (b) Graphene (and graphite) thickness vs initial a-C thickness ${ }^{[31]}$

Samples were annealed at $800{ }^{\circ} \mathrm{C}$ for 15 min with a $300 \mathrm{~nm}$ Ni catalyst layer
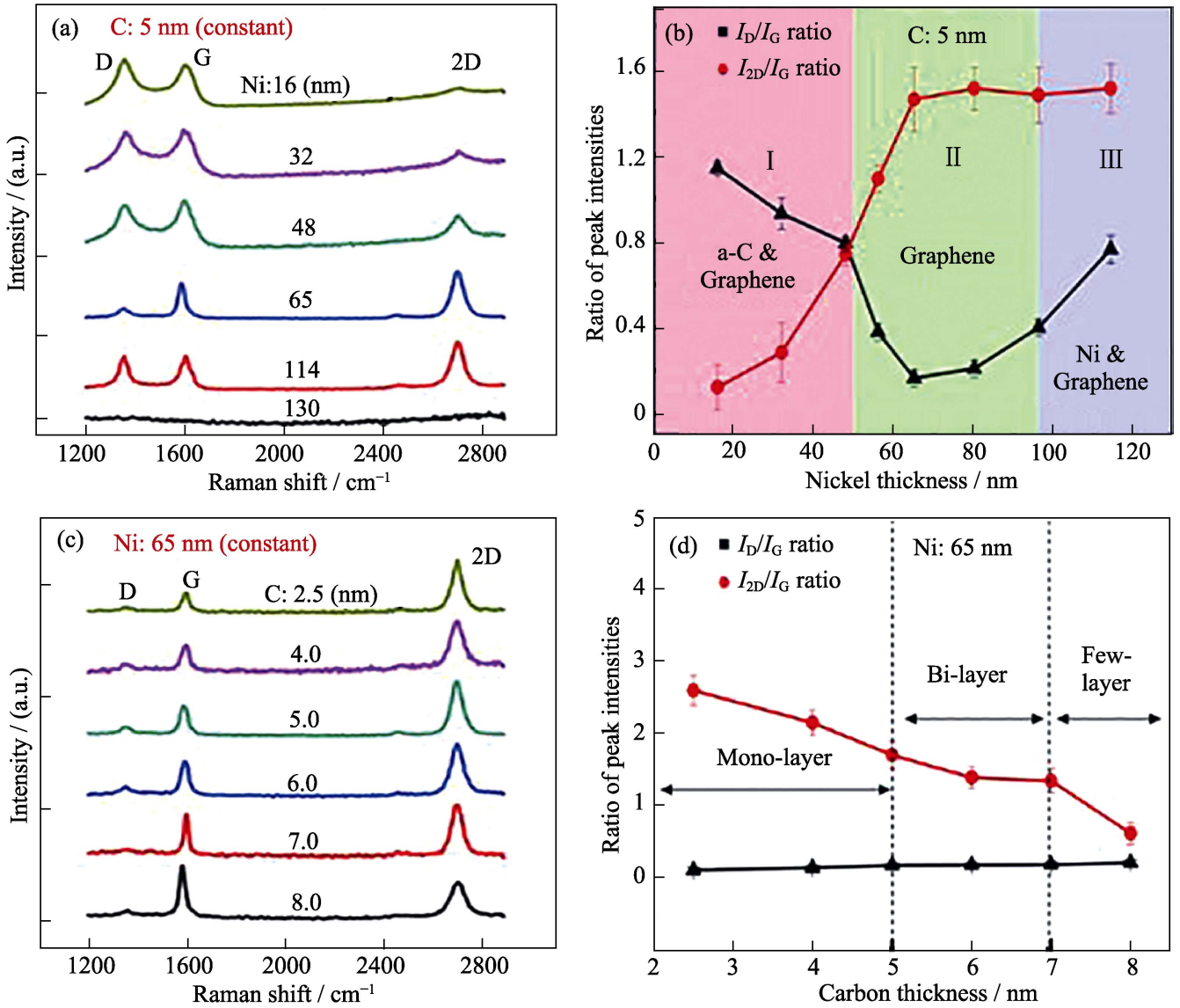

图 $9 \mathrm{a}-\mathrm{C}$ 和 $\mathrm{Ni}$ 厚度对石墨烯生长的影响 ${ }^{[46]}$

Fig. 9 Influence of $\mathrm{Ni}$ and $\mathrm{C}$ film thicknesses on graphene growth ${ }^{[46]}$

(a) Raman spectra of RTP graphene grown with a $5 \mathrm{~nm} \mathrm{C}$ film covered with a Ni film of different thicknesses; (b) $I_{\mathrm{D}} / I_{\mathrm{G}}$ and $I_{2 \mathrm{D}} / I_{\mathrm{G}}$ Raman peak ratios of the RTP graphene as functions of Ni film thickness; (c) Raman spectra of RTP graphene grown with a $65 \mathrm{~nm}$ Ni film on top of a C film with different thicknesses; (d) $I_{\mathrm{D}} / I_{\mathrm{G}}$ and $I_{2 \mathrm{D}} / I_{\mathrm{G}}$ Raman peak ratios of the RTP graphene as functions of C film thickness

度以及晶面取向。金属 $\mathrm{Ni}$ 的(111)晶面与石墨的(002) 晶格适配度很小 ${ }^{[44,49]}$, 因此很多研究者致力于提高 $\mathrm{Ni}$ 的取向性, 以期获得质量良好的石墨烯。Orofeo 等 ${ }^{[40]}$ 用溅射法分别在室温和 $500^{\circ} \mathrm{C}$ 沉积 $\mathrm{Ni}$ 层, 发现
高温下得到的 Ni 层结晶性更好，且具有(111)择优 取向，进而制备质量更优异的石墨烯。Kwak 等 ${ }^{[50]}$ 通过电子束蒸发的方式沉积 $\mathrm{Ni}$ 层, 并对 $\mathrm{Ni}$ 层进行 $1000^{\circ} \mathrm{C}$ 热处理，同样得到(111)择优取向的 $\mathrm{Ni}$ 催化 
层, 最终也得到高质量的单层石墨烯。此外, 研究学 者们还发现表面越平整的 $\mathrm{Ni}$ 得到的石墨烯质量越 高 ${ }^{[44]}$ 。退火时间也会影响石墨烯的生成质量。Zheng 等 ${ }^{[31]}$ 发现, 在催化温度范围内, 退火时间对石墨烯 没有明显的影响，但在低于催化温度时，退火时间 对石墨烯的生成有明显影响。本课题组也发现, 即 使是在低温下，只要经过足够长时间退火，亦有部 分非晶碳发生了石墨化转变, 生成多层石墨烯 ${ }^{[51]}$ 。

除此之外, 退火气氛亦对生成的石墨烯有所影 响。Asaka 等 ${ }^{[49]}$ 发现, 在高真空度下 $\mathrm{Ni}$ 未被氧化, 可 自发催化非晶碳进行石墨化转变。但在大气中被氧 化后的 $\mathrm{Ni}$ 催化性能明显下降, 需要提高退火温度才 能生成石墨烯。如果在退火过程中通入氢气, 生成 的石墨烯质量会更好 ${ }^{[30]}$ 。这是因为通入氢气使石墨 烯易于形核, 并避免其表面氧化 ${ }^{[52]}$ 。Chen 等 ${ }^{[53]}$ 发现 高温下氢气既可以使非晶碳发生转变生成石墨烯, 也可以对过量的非晶碳产生刻蚀作用。

\section{2 催化机理}

金属催化非晶碳转化石墨烯的研究主要集中在 工艺探索方面, 对其生长机理研究甚少, 且说法不 一, 目前被普遍接受的是溶解-析出机制 ${ }^{[34,36]}$ 。即在 高温下, $\mathrm{C}$ 原子首先扩散进入金属催化剂层 $(\mathrm{Ni} 、 \mathrm{Co}$ 等)中, 低温时 $\mathrm{C}$ 再以 $\mathrm{sp}^{2}$ 形式析出, 并在表面形核 生长成石墨烯。Rodríguez 等 ${ }^{[25]}$ 利用 STEM 观测到 非晶碳在加热过程中金属发生熟化, 之后析出石墨 烯。这是扩散机制促进了石墨烯的生成, 当 $\mathrm{C}$ 溶解 进入 $\mathrm{Ni}$ 之后, 再从金属 $\mathrm{Ni}$ 中析出形成石墨烯, 如图 10 所示。Rodríguez 等 ${ }^{[25]}$ 与 Schneider 等 ${ }^{[54]}$ 对非晶碳
的溶解和析出进行了详细阐述, 认为发生非晶碳转 化成石墨烯是因为非晶碳的能量高于石墨烯，所以 析出时碳原子倾向于形成石墨烯，从而以更稳定的 形式存在。

还有研究者提出晶界偏析 ${ }^{[5]}$ 或金属诱导 ${ }^{[41]}$ 等 不同的观点。Orofeo 等 ${ }^{[40]}$ 通过实验证明, 在(111)择 优取向的 $\mathrm{Ni}$ 金属层催化下，可以得到高质量的单层 石墨烯; 而得不到结晶性差的 $\mathrm{Ni}$, 这说明晶界减少 导致碳的形核位点减少，而 $\mathrm{C}$ 原子通过晶界进行扩 散也就更少，从而可以形成质量更好的石墨烯。即 对于晶界偏析而言, 晶界的多少对生成的石墨烯影 响至关重要。Kwak 等 ${ }^{[50]}$ 则对不同温度下的扩散原 理进行了研究, 发现在低于 $260^{\circ} \mathrm{C}$ 时, $\mathrm{C}$ 原子优先在 $\mathrm{Ni}$ 的晶界处扩散; 而温度升高后, 体相扩散逐渐增 大，诱发形成多层石墨烯，这表明石墨烯转化受晶 界偏析和溶解-析出机制共同影响，并因退火温度 的不同而具有不同的主导作用。另外, Saenger 等 ${ }^{[41]}$ 利用原位 XRD 发现在 $630^{\circ} \mathrm{C}$ 下就出现了石墨的(002) 峰, 如图 7 所示, 并且低温下 $\mathrm{C}$ 在 $\mathrm{Ni}$ 中的溶解度很 低, 因此不能简单地归因为溶解沉积原理。在高温 条件下出现石墨的(002)峰, 说明高温下 $\mathrm{a}-\mathrm{C}$ 发生了 转变, 这应该是金属诱导机理, 与 $\mathrm{Al}$ 诱导非晶硅类 似。这与溶解一析出区别在于, 溶解析出是在降温阶 段析出石墨烯(图 11), 而金属催化则是达到催化反 应温度后即可得到石墨烯。

实质上无论是溶解一析出、金属诱导或者晶界偏 析机制，本质上均与 $\mathrm{C}$ 在金属中的溶解度息息相 关。以上研究文献已经证实, 低 $\mathrm{C}$ 溶解度的金属如 $\mathrm{Cu}$ 难以催化非晶碳得到石墨烯，而具有一定 $\mathrm{C}$ 溶解 度的 $\mathrm{Ni} 、 \mathrm{Co}$ 等金属则可以催化非晶碳生成石墨烯。
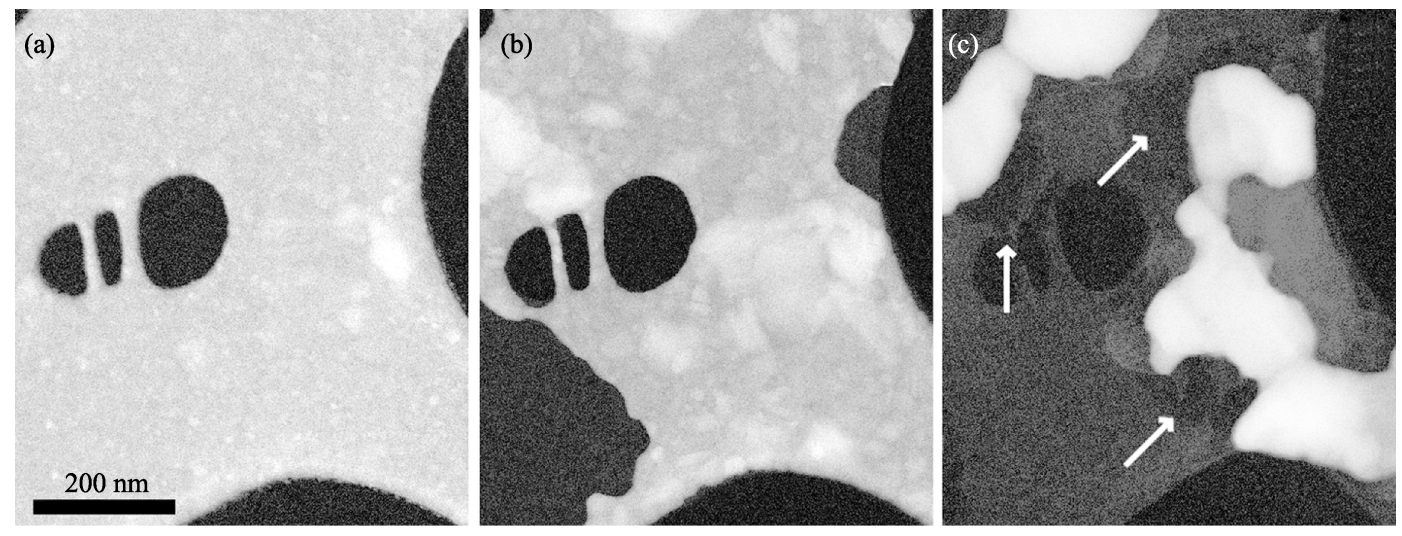

图 10 非晶碳上 Ni 的暗场 STEM 照片 ${ }^{[25]}$

Fig. 10 Plan-view scanning transmission electron microscopy (STEM) dark-field images of Ni crystals on an amorphous carbon film at $400^{\circ} \mathrm{C}$

(a), $600^{\circ} \mathrm{C}$

(b) and $720^{\circ} \mathrm{C}$

(c) ${ }^{[25]}$

(a) At $400^{\circ} \mathrm{C}$, the coherent polycrystalline Ni film (bright) covers the $\mathrm{C}$ substrate entirely; holes in the $\mathrm{C}$ film appear black; (b) At $600^{\circ} \mathrm{C}$,

ripening of the metal crystals starts and uncovers areas of the amorphous carbon film (light gray contrast); (c) At $720^{\circ} \mathrm{C}$,

ripening continues and graphene areas appear (dark, marked with arrows) 


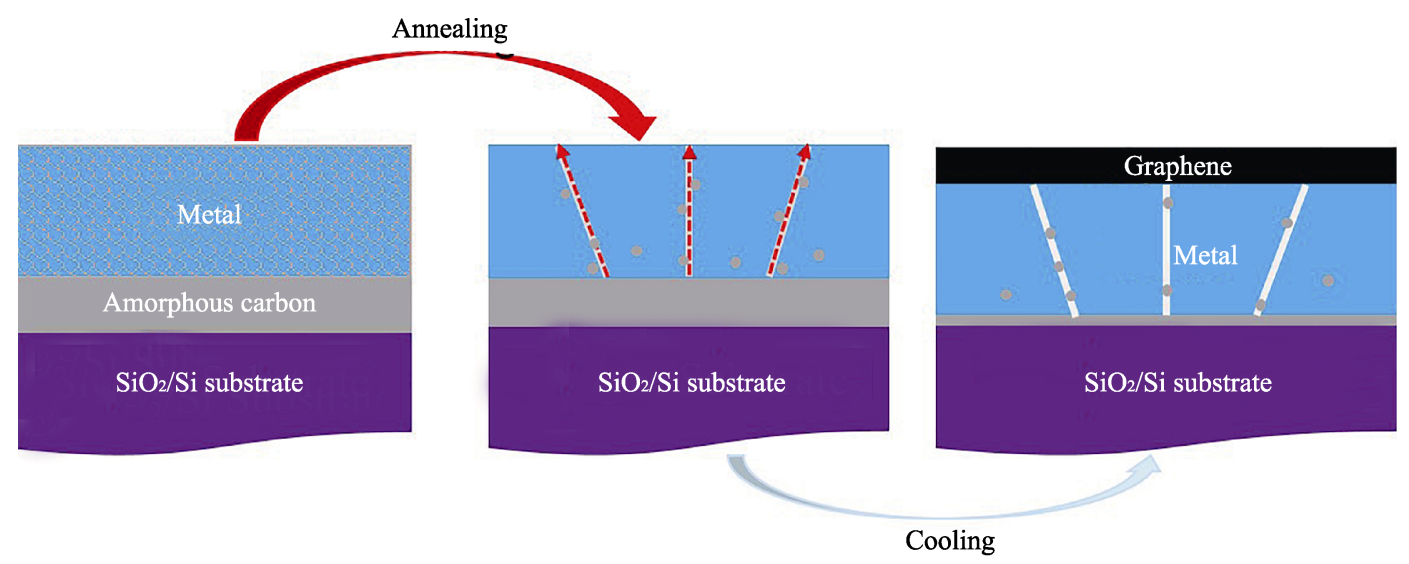

图 11 溶解析出形成石墨烯的示意图

Fig. 11 Schematic illustration of graphene formation via $\mathrm{C}$ dissolution-precipitation

因此，欲揭示金属催化石墨烯的生长机制，最重要 是需要阐明 $\mathrm{C}$ 在金属中的扩散行为。然而, 目前扩散 机理仍不明确, 有待澄清, 亟待进一步的深入研究。

\section{3 结束语}

非晶碳与石墨烯互为同素异形体, 用其作为固 体碳源前驱体制备石墨烯是一种新颖的方法。相比 于采用气体碳源的 CVD 方法, 利用非晶碳热退火方 法, 可以在低温条件下、多种基底材料(陶瓷、金属 等)上大面积制备石墨烯。但是碳膜种类、金属催化 层种类、碳膜/金属层比例、热处理工艺等诸多因素 的影响, 国内外对于非晶碳转化形成石墨烯的晶态 结构转变、碳源的扩散规律、以及动力学生长机制 方面还不明确, 亟需深入研究。从可控制备角度来看, 一方面需要进一步优化工艺, 实现层数、尺寸可控的 高质量石墨烯的稳定生长; 另一方面, 需要研究和 发展可靠性高的石墨烯表征方法, 特别是对于非晶 碳在金属层下方的结构体系，如果石墨烯中复合有 非晶碳成分, 则对传统拉曼光谱的测量精确度提出 挑战。另外, 在理论研究方面, 由于影响非晶碳转变 石墨烯的因素颇多, 如何实现非晶碳制备高质量石 墨烯关键参数的篎选和优化设计, 并从原子和分子 角度深入理解非晶碳到石墨烯过程中的晶态调控 机理、石墨烯生长机制等, 也是迫切需要开展的重要 工作。

\section{参考文献:}

[1] BUNDY F P, HAll H T, STRONG H M, et al. Man-made diamonds. Nature, 1955, 176(4471): 51-55.

[2] AISENBERG S, CHABOT R. Ion-beam deposition of thin films of diamondlike carbon. Journal of Applied Physics, 1971, 42(7): 2953-2958.

[3] KROTO H W, HEATH J R, O'Brien S C, et al. C60: bucminster- fuleene. Nature, 1985, 318(6042): 162-163.

[4] LIJIMA SUMIO. Helical microtubules of graphitic carbon. Nature, 1991, 354(6348): 56-58.

[5] NOVOSELOV K S, GEIM A K, MOROZOV S V, et al. Electric field effect in atomically thin carbon films. Science, 2004, 306(5696): 666-669.

[6] LI G, LI Y, LIU H, et al. Architecture of graphdiyne nanoscale films. Chemical Communications, 2010, 46(19): 3256-3258.

[7] LEE C, WEI X, KYSAR J W, et al. Measurement of the elastic properties and intrinsic strength of monolayer graphene. Science, 2008, 321(5887): 385-388.

[8] NAIR R R, BLAKE P, GRIGORENKO A N, et al. Fine structure constant defines visual transparency of graphene. Science, 2008, 320(5881): 1308.

[9] BALANDIN A A, GHOSH S, BAO W, et al. Superior thermal conductivity of single layer graphene. Nano Letters, 2008, 8(3): 902- 907.

[10] MOROZOV S V, NOVOSELOV K S, KATSNELSON M I, et al. Giant intrisic carrier mobilities in graphene and its bilayer. Physical Review Letters, 2008, 100(1): 016602.

[11] OHNO Y, MAEHASHI K, YAMASHIRO Y, et al. Electrolytegated graphene field-effect transistors for detecting $\mathrm{pH}$ and protein adsorption. Nano Letters, 2009, 9(9): 3318-3322.

[12] YADAV P, BANERJEE A, UNNI S, et al. A 3D hexaporous carbon assembled from single-layer graphene as high performance supercapacitor. ChemSusChem, 2012, 5(11): 2159-2164.

[13] LI S S, TU K H, LIN C C, et al. Solution-processable graphene oxide as an efficient hole transport layer in polymer solar cells. ACS Nano, 2010, 4(6): 3169-3174.

[14] LIN Y M, DIMITRAKOPOULOS C, JENKINS K A, et al. $100-\mathrm{GHz}$ transistors from wafer scale epitaxial graphene. Science, 2010, 327(5966): 662 .

[15] EMTSEV K V, BOSTWICK A, HORN K, et al. Towards wafersize graphene layers by atmospheric pressure graphitization of silicon carbide. Nature Materials, 2009, 8(3): 203-207.

[16] WARNER J H, SCHAFFEL F, RUMMELI M, et al. Graphene: Fundamentals and Emergent Applications. Boston: Newnes, 2012: 204-213.

[17] PARK S, RUOFF R S. Chemical methods for the production of graphenes. Nature Nanotechnology, 2009, 4(4): 217-224.

[18] STANKOVICH S, DIKIN D A, PINER R D, et al. Synthesis of graphene-based nanosheets via chemical reduction of exfoliated graphite oxide. Carbon, 2007, 45(7): 1558-1565. 
[19] LI X, CAI W, AN J, et al. Large-area synthesis of high-quality and uniform graphene films on copper foils. Science, 2009, 324(5932): 1312-1314.

[20] 任文才, 高力波, 马来鹏, 等. 石墨烯的化学气相沉积法制备. 新型碳材料, 2011, 26(01): 71-80.

[21] 马来鹏, 任文才, 董再励, 等. 铜表面化学气相沉积石墨烯的研 究进展: 生长行为与控制制备. 科学通报, 2012, 57(23): 21582163.

[22] KANG J, SHIN D, BAE S, et al. Graphene transfer: key for applications. Nanoscale, 2012, 4(18): 5527-5537.

[23] SUN Z, YAN Z, YAO J, et al. Growth of graphene from solid carbon sources. Nature, 2010, 468(7323): 549-552.

[24] YAN Z, PENG Z, SUN Z, et al. Growth of bilayer graphene on insulating substrates. ACS Nano, 2011, 5(10): 8187-8192.

[25] RODRIGUEZ-MANZO J A, PHAM-HUU C, BANHART F. Graphene growth by a metal catalyzed solid-state transformation of amorphous carbon. ACS Nano, 2011, 5(2): 1529-1534.

[26] 王茂章, 杨全红, 成会明. 碳的结构及其同素异性体. 炭素技术, 2001(1): 23-28.

[27] BAI L, ZHANG G, LU Z, et al. Tribological mechanism of hydrogenated amorphous carbon film against pairs: a physical description. Journal of Applied Physics, 2011, 110(3): 033521.

[28] ROBERTSON J. Diamond-like amorphous carbon. Materials Science and Engineering: R: Reports, 2002, 37(4): 129-281.

[29] DONNET C, ERDEMIR A. Historical developments and new trends in tribological and solid lubricant coatings. Surface and Coatings Technology, 2004, 180: 76-84.

[30] KOSHIDA K, GUMI K, OHNO Y, et al. Position-controlled direct graphene synthesis on silicon oxide surfaces using laser irradiation. Applied Physics Express, 2013, 6(10): 105101.

[31] ZHENG M, TAKEI K, HSIA B, et al. Metal-catalyzed crystallization of amorphous carbon to graphene. Applied Physics Letters, 2010, 96(6): 063110.

[32] SEO J H, LEE H W, KIM J K, et al. Few layer graphene synthesized by filtered vacuum arc system using solid carbon source. Current Applied Physics, 2012, 12: 131-133.

[33] PENG Z W, YAN Z, SUN Z Z, et al. Direct growth of bilayer graphene on $\mathrm{SiO}_{2}$ substrates by carbon diffusion through nickel. ACS Nano, 2011, 5(10): 8241-8247.

[34] LI X, CAI W, COLOMBO L, et al. Evolution of graphene growth on $\mathrm{Ni}$ and $\mathrm{Cu}$ by carbon isotope labeling. Nano Letters, 2009, 9(12): 4268-4272.

[35] MIYOSHI M, MIZUNO M, BANNO K, et al. Study on transferfree graphene synthesis process utilizing spontaneous agglomeration of catalytic Ni and Co metals. Materials Research Express, 2015, 2(1): 015602.

[36] FUJITA J, UEKI R, MIYAZAWA Y, et al. Graphitization at interface between amorphous carbon and liquid gallium for fabricating large area graphene sheets. Journal of Vacuum Science \& Technology B, 2009, 27(6): 3063-3066.

[37] HATAKEYAMA T, KOMETANI R, WARISAWA S, et al. Selective graphene growth from DLC thin film patterned by focusedion-beam chemical vapor deposition. Journal of Vacuum Science \& Technology B, 2011, 29(6): 06FG04.

[38] WANG J, CHEN L F, WU N, et al. Uniform graphene on liquid metal by chemical vapour deposition at reduced temperature. Carbon, 2016, 96: 799-804.

[39] HIRANO R, MATSUBARA K, KALITA G, et al. Synthesis of transfer-free graphene on an insulating substrate using a solid phase reaction. Nanoscale, 2012, 4(24): 7791-7796.

[40] OROFEO C M, AGO H, HU B, et al. Synthesis of large area, homogeneous, single layer graphene films by annealing amorphous carbon on Co and Ni. Nano Research, 2011, 4(6): 531-540.

[41] SAENGER K L, TSANG J C, BOL A A, et al. In situ X-ray diffraction study of graphitic carbon formed during heating and cooling of amorphous-C/Ni bilayers. Applied Physics Letters, 2010, 96(15): 153105.

[42] BANNO K, MIZUNO M, FUJITA K, et al. Transfer-free graphene synthesis on insulating substrates via agglomeration phenomena of catalytic nickel films. Applied Physics Letters, 2013, 103(8): 082112.

[43] CHU J H, KWAK J, KWON T Y, et al. Facile synthesis of fewlayer graphene with a controllable thickness using rapid thermal annealing. ACS Applied Materials \& Interfaces, 2012, 4(3): 1777 1782.

[44] WENISCH R, HÜBNER R, MUNNIK F, et al. Nickel-enhanced graphitic ordering of carbon ad-atoms during physical vapor deposition. Carbon, 2016, 100: 656-663.

[45] ANTON R. On the reaction kinetics of Ni with amorphous carbon. Carbon, 2008, 46(4): 656-662.

[46] XIONG W, ZHOU Y S, JIANG L J, et al. Single-step formation of graphene on dielectric surfaces. Advanced Materials, 2013, 25(4): 630-634.

[47] LENG Y, XIE L, LIAO F, et al. Kinetic and thermodynamics studies on the decompositions of $\mathrm{Ni}_{3} \mathrm{C}$ in different atmospheres. Thermochimica Acta, 2008, 473(1): 14-18.

[48] KOVÁCS G J, BERTÓTI I, RADNÓCZI G. X-ray photoelectron spectroscopic study of magnetron sputtered carbon-nickel composite films. Thin Solid Films, 2008, 516(21): 7942-7946.

[49] ASAKA K, SAITO Y. Spontaneous graphenization of amorphous carbon on clean surfaces of nanometer-sized nickel particles at room temperature. Carbon, 2016, 103: 352-355.

[50] KWAK J, CHU J H, CHOI J K, et al. Near room-temperature synthesis of transfer-free graphene films. Nature Communications, 2012, 3: 645 .

[51] 刘盼盼, 李汉超, 杨 林 等. 退火温度对金属催化四面体非晶 碳转变石墨过程的影响. 材料研究学报, 2018. DOI:10.11901/ 1005.3093.2017.107.

[52] NGUYEN B S, LIN J F, PERNG D C. Non-vacuum growth of graphene films using solid carbon source. Applied Physics Letters, 2015, 106(22): 221604.

[53] CHEN Y Z, MEDINA H, LIN H C, et al. Large-scale and patternable graphene: direct transformation of amorphous carbon film into graphene/graphite on insulators via $\mathrm{Cu}$ mediation engineering and its application to all-carbon based devices. Nanoscale, 2015, 7(5): 1678-1687.

[54] SCHNEIDER J J. Transforming amorphous into crystalline carbon: observing how graphene grows. ChemCatChem, 2011, 3(7): 1119-1120.

[55] 张朝华, 付 否, 张艳锋, 等. 石墨烯催化生长中的偏析现象及 其调控方法. 化学学报, 2013, 71(03): 308-322. 